\title{
A QUANTUM FIELD THEORETICAL REPRESENTATION OF EULER-ZAGIER SUMS
}

\author{
UWE MÜLLER and CHRISTIAN SCHUBERT
}

Received 4 June 2001 and in revised form 16 January 2002

\begin{abstract}
We establish a novel representation of arbitrary Euler-Zagier sums in terms of weighted vacuum graphs. This representation uses a toy quantum field theory with infinitely many propagators and interaction vertices. The propagators involve Bernoulli polynomials and Clausen functions to arbitrary orders. The Feynman integrals of this model can be decomposed in terms of a vertex algebra whose structure we investigate. We derive a large class of relations between multiple zeta values, of arbitrary lengths and weights, using only a certain set of graphical manipulations on Feynman diagrams. Further uses and possible generalisations of the model are pointed out.
\end{abstract}

2000 Mathematics Subject Classification: 05A19, 11M06, 81T18.

1. Introduction. The perturbative evaluation of Green's functions in quantum field theory leads to a class of iterated parameter integrals whose explicit calculation becomes very difficult beyond the first few orders in the coupling constant expansion. Any progress in this area of work must be based on an intimate knowledge of the properties of various types of special functions such as polylogarithms, hypergeometric functions, and their generalisations (see [39]).

In recent years, some structure is seen to emerge from the seemingly haphazard occurrence of those special functions as the values of individual Feynman diagrams. Kreimer's hypothesis [27], based on a rule of associating knots to Feynman diagrams, allows one to predict from knot-theoretical considerations the level of transcendentality which can possibly appear in the counterterm coefficients of an ultraviolet divergent diagram. Even though it has been verified for a large number of examples [13] the raison d'être for the correspondence between graphs and knots remains presently mysterious. More recently, there are indications that knot-theoretical concepts may be of relevance even for the finite parts of Feynman diagrams [11].

The remarkably rich mathematical structures surfacing in this correspondence make Feynman diagrams increasingly interesting from the pure mathematician's point of view. The objects encountered in the calculation of UV divergences in perturbative quantum field theory, multiple harmonic sums, are of considerable relevance to number theory and other branches of mathematics (see [15, 17, 19, 28, 34, 41, 42, 44]).

Quantum field theory amplitudes can be calculated in coordinate space or in momentum space. In four-dimensional field theory the arising integrals are normally of a similar type and degree of difficulty. This is very different in the case of a onedimensional quantum field theory compactified on a circle, which is considered in the present paper. Such quantum field theories arise naturally if one represents one-loop 
amplitudes in $D$-dimensional field theory in terms of first-quantized path integrals. An approach to quantum field theory along these lines has gained some popularity in recent years after it was discovered that it allows one to reorganise ordinary field theory amplitudes in a manner similar to string theory amplitudes [4, 33, 37, 38, 40]. In this type of formalism the $D$-dimensional space-time enters as a target space, and amplitudes are calculated in terms of an auxiliary field theory in one-dimensional parameter space. Green's functions in parameter space are then used for the evaluation of Feynman diagrams in this one-dimensional worldloop theory.

As a simple example, consider the one-loop effective action for a scalar field theory with a $\left(\lambda / 3\right.$ !) $\phi^{3}$ interaction. This effective action can be expressed in terms of a firstquantized path integral as follows:

$$
\Gamma[\phi]=\frac{1}{2} \int_{0}^{\infty} \frac{d T}{T} e^{-m^{2} T} \int_{x(T)=x(0)} \mathscr{D} x(\tau) e^{-\int_{0}^{T} d \tau\left((1 / 4) \dot{x}^{2}+\lambda \phi(x(\tau))\right)} .
$$

Here, $T$ is the usual Schwinger proper-time for the particle circulating in the loop. At fixed $T$ a path integral has to be calculated over the space of closed loops in spacetime with period $T$. This integral contains a zero mode which is removed by fixing the center-of-mass of the loop $x_{0} \equiv(1 / T) \int_{0}^{T} d \tau x(\tau)$. The reduced path integral is evaluated perturbatively by expanding the interaction exponential and using the parameter space Green's function

$$
G\left(\tau_{1}, \tau_{2}\right) \equiv 2 T \sum_{\substack{n=-\infty \\ n \neq 0}}^{\infty} \frac{e^{2 \pi i n\left(\tau_{1}-\tau_{2}\right) / T}}{(2 \pi i n)^{2}}=\left|\tau_{1}-\tau_{2}\right|-\frac{\left(\tau_{1}-\tau_{2}\right)^{2}}{T}-\frac{T}{6} .
$$

(The constant part of this Green's function is irrelevant for the final physical results and usually deleted from the beginning.) Momentum space methods have also sometimes been used, which in this case lead to Fourier sum representations. In [3, 14], a number of such sums were calculated to provide a check on the resolution of certain ambiguities which in the first-quantized formalism can arise in curved backgrounds. In this comparison we find that terms given by simple polynomial integrals in coordinate space may, in momentum space, correspond to nontrivial multiple sums of the Euler-Zagier type.

In the present work we turn the logic around, and use this formalism as a tool for the systematic study of Euler-Zagier sums. Euler-Zagier sums, also called multiple $\zeta$ values or multiple harmonic series, are defined by

$$
\zeta\left(k_{1}, \ldots, k_{m}\right)=\sum_{n_{1}>n_{2}>\cdots>n_{m}>0} \frac{1}{n_{1}^{k_{1}} \cdots n_{m}^{k_{m}}}=\operatorname{Li}_{k_{1} \cdots k_{m}}(1, \ldots, 1) .
$$

They are special values of the multidimensional polylogarithms $\mathrm{Li}_{k_{1}, \ldots, k_{m}}$, defined as

$$
\operatorname{Li}_{k_{1}} \cdots k_{m}\left(z_{1}, \ldots, z_{m}\right) \equiv \sum_{n_{1}>n_{2}>\cdots>n_{m}>0} \frac{z_{1}^{n_{1}} \cdots z_{m}^{n_{m}}}{n_{1}^{k_{1}} \cdots n_{m}^{k_{m}}} .
$$

We call $m$ the length (or depth) of such a series, and $k_{1}+k_{2}+\cdots+k_{m}$ its level (or weight). Sums of the type (1.3) were first considered by Euler [16]. Euler himself noted 
that numerous relations exist between Euler-Zagier sums. Some simple examples are the following (all given by Euler):

$$
\begin{aligned}
& \zeta(2,1)=\zeta(3), \\
& \zeta(3,1)=\frac{3}{2} \zeta(4)-\frac{1}{2} \zeta^{2}(2)=\frac{\pi^{4}}{360}, \\
& \zeta(2,2)=\frac{1}{2} \zeta^{2}(2)-\frac{1}{2} \zeta(4)=\frac{\pi^{4}}{120} \\
& \zeta(3,2)=-\frac{11}{2} \zeta(5)+3 \zeta(2) \zeta(3), \\
& \zeta(4,1)=2 \zeta(5)-\zeta(2) \zeta(3) .
\end{aligned}
$$

Further results for the length-two case can be found in [1, 43]. Systematic investigations of Euler-Zagier sums of length higher than two have been undertaken only in recent years [2, 6, 9, 20, 21, 22, 24, 25, 31, 32, 35]. From the point of view of physics, the study of their relations is relevant for attempts at a classification of the possible ultraviolet divergences in quantum field theory [12, 13].

We would like to be able to represent arbitrary such sums in terms of one-dimensional Feynman diagrams. To achieve this goal we have to generalise the usual worldline path integral formalism in the following ways:

(1) As explained above, the first-quantized loop path integral is defined to run over the space of all periodic functions, with the constant functions eliminated. Here we will restrict it to one "chiral half" spanned by the basis functions $f_{n}(u)=e^{2 \pi i n u}$, $n=1,2, \ldots$ (This amounts to a complexification of spacetime.)

(2) We choose the kinetic term of our model in such a way that arbitrary inverse powers of derivatives will appear.

Those purely mathematical considerations lead us to define the $\zeta$-model, a onedimensional quantum field theory given by the following partition function,

$$
\begin{gathered}
Z(g, \lambda)=\int_{\mathscr{H}} \mathscr{D} x(u) e^{-S}, \\
S=\int_{0}^{1} d u_{1} \int_{0}^{1} d u_{2} \frac{1}{2} \bar{x}\left(u_{1}\right)\left(I-\lambda 2 \pi i \partial^{-1}\right) x\left(u_{2}\right)-\int_{0}^{1} d u e^{g x(u)+\bar{g} \bar{x}(u)} .
\end{gathered}
$$

Here and in the following $\partial \equiv(d / d u)$ denotes the ordinary derivative. The path integral is to be performed over the Hilbert space

$$
\mathscr{H}=\left\{\left.x(u)\left|x(u)=\sum_{n=1}^{\infty} a_{n} e^{2 \pi i n u}, \sum_{n=1}^{\infty}\right| a_{n}\right|^{2}<\infty\right\} .
$$

The perturbative expansion of both the kinetic and the interaction terms for this toy model leads to the Feynman rules represented in Figure 1.1.

In Section 2, we write the propagators $g_{12}^{(k)}$ explicitly in terms of Bernoulli polynomials and Clausen functions. We show that any multiple $\zeta$ sum as in (1.3) can be represented as a Feynman diagram in this model. In Section 3, we investigate the properties of the elementary tree-level $n$-point integrals with arbitrary external propagators. Section 4 demonstrates how one can use partial integrations and reality conditions to derive a large class of relations between multiple $\zeta$ sums. In Section 5 , we point out possible further uses of the model, as well as some generalisations. 


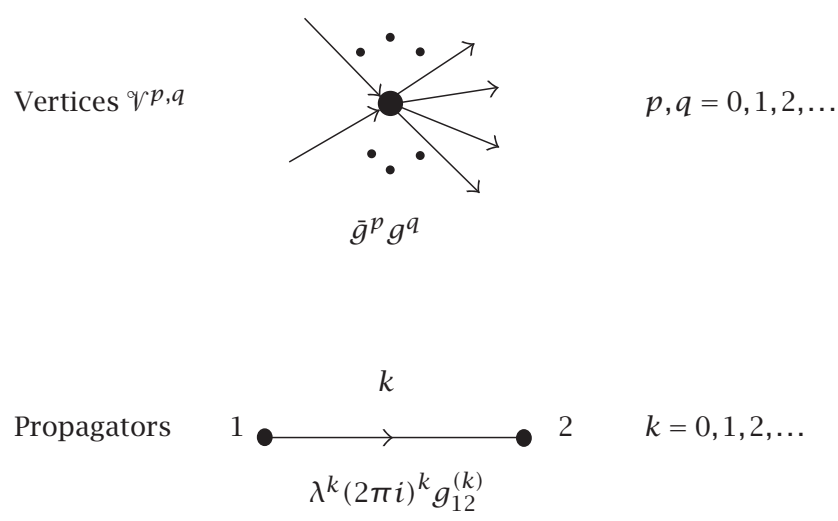

FIGURE 1.1. Feynman rules of the $\zeta$-model.

2. Basic properties. Inverting the kinetic part of our (nonlocal) Lagrangian (1.6), and writing $\partial^{-k}$ in the defining basis of the Hilbert space $\mathscr{H}$, we find

$$
g_{12}^{(k)} \equiv g^{(k)}\left(u_{12}\right)=\sum_{n=1}^{\infty} \frac{e^{2 \pi i n u_{12}}}{(2 \pi i n)^{k}} .
$$

If we represent the unit circle in the complex plane, this sum will turn into the $k$ th polylogarithm,

$$
g_{12}^{(k)}=\frac{1}{(2 \pi i)^{k}} \operatorname{Li}_{k}\left(\frac{z_{1}}{z_{2}}\right)
$$

$\left(u_{12}=u_{1}-u_{2}, z_{i}=e^{2 \pi i u_{i}}\right)$. We note the following properties of $g^{(k)}$ :

$$
\begin{gathered}
\frac{\partial}{\partial u_{1}} g_{12}^{(k)}=-\frac{\partial}{\partial u_{2}} g_{12}^{(k)}=g_{12}^{(k-1)}, \\
g_{21}^{(k)}=(-1)^{k} \bar{g}_{12}^{(k)}=g^{(k)}\left(1-u_{12}\right), \\
g^{(k)}(0)=\frac{\zeta(k)}{(2 \pi i)^{k}}, \\
\int_{0}^{1} d u_{1,2} g_{12}^{(k)}=0 .
\end{gathered}
$$

Equation (2.3) can be inverted using the explicitly known integral kernel for inverse derivatives in this space [37],

$$
\begin{aligned}
g_{12}^{(k+l)} & =\int_{0}^{1} d u\left\langle u_{1}\left|\partial^{-l}\right| u\right\rangle g^{(k)}\left(u-u_{2}\right), \\
\left\langle u_{1}\left|\partial^{-n}\right| u_{2}\right\rangle & =-\frac{1}{n !} B_{n}\left(\left|u_{12}\right|\right) \operatorname{sign}^{n}\left(u_{12}\right) \\
& =-\frac{B_{n}\left(u_{12}\right)}{n !}+\frac{u_{12}^{n-1}}{2(n-1) !}\left(\operatorname{sign}\left(u_{12}\right)-1\right) .
\end{aligned}
$$

Here $B_{k}$ denotes the $k$ th Bernoulli polynomial. To write $g_{12}^{(k)}$ more explicitly we split it into its real and imaginary parts,

$$
g_{12}^{(k)}=\overline{\mathfrak{g}}_{12}^{(k)}+i \hat{\mathfrak{g}}_{12}^{(k)} .
$$


Using the standard integral representation of the polylogarithm

$$
\mathrm{Li}_{k}(z)=\frac{(-1)^{k-1}}{(k-1) !} z \int_{0}^{1} d x \frac{\ln ^{k-1}(x)}{1-x z},
$$

it is then easy to show that

$$
\begin{aligned}
g_{12}^{(0)}= & \frac{1}{2}\left(\delta\left(u_{12}\right)-1+i \cot \left(\pi u_{12}\right)\right), \\
g_{12}^{(1)}= & \frac{1}{2}\left(\frac{1}{2} \operatorname{sign}\left(u_{12}\right)-u_{12}+\frac{i}{\pi} \ln \left|2 \sin \left(\pi u_{12}\right)\right|\right), \\
g_{12}^{(2)}= & \frac{1}{4}\left(\left|u_{12}\right|-u_{12}^{2}-\frac{1}{6}+\frac{i}{\pi^{2}} \int_{0}^{1} d \xi \frac{\ln \xi \sin \left(2 \pi u_{12}\right)}{1-2 \xi \cos \left(2 \pi u_{12}\right)+\xi^{2}}\right), \\
g_{12}^{(3)}= & -\frac{1}{24}\left(\operatorname{sign}\left(u_{12}\right)-2 u_{12}\right)\left(\left|u_{12}\right|-u_{12}^{2}\right) \\
& +\frac{i}{16 \pi^{3}} \int_{0}^{1} \frac{d \xi}{\xi} \ln \xi \ln \left(1-2 \xi \cos \left(2 \pi u_{12}\right)+\xi^{2}\right), \\
g_{12}^{(k \neq 0 \text { even })=} & -\frac{1}{2} \frac{1}{k !} B_{k}\left(\left|u_{12}\right|\right) \\
& +\frac{i}{(2 \pi)^{k}} \frac{(-1)^{(k / 2)+1}}{(k-1) !} \int_{0}^{1} d \xi \ln ^{k-1} \xi \frac{\sin \left(2 \pi u_{12}\right)}{1-2 \xi \cos \left(2 \pi u_{12}\right)+\xi^{2}}, \\
g_{12}^{(k \neq 1 \text { odd })}= & -\frac{1}{2} \frac{1}{k !} B_{k}\left(\left|u_{12}\right|\right) \operatorname{sign}\left(u_{12}\right) \\
& +\frac{i}{2(2 \pi)^{k}} \frac{(-1)^{(k+1) / 2}}{(k-2) !} \int_{0}^{1} \frac{d \xi}{\xi} \ln ^{k-2} \xi \ln \left(1-2 \xi \cos \left(2 \pi u_{12}\right)+\xi^{2}\right),
\end{aligned}
$$

$\left(u_{12} \in[-1,1]\right)$. Note also that the imaginary part of $g_{12}^{(k)}$ is, up to a normalisation factor, identical with the $k$ th Clausen function $\mathrm{Cl}_{k}\left(2 \pi u_{12}\right)$ (see [29, 30]). Only the real parts are present in the calculation of worldline path integrals in standard field theory. In particular, note that $\bar{g}^{(2)}$ is, up to a conventional factor of 4 , identical with the function $G$ introduced in $(1.2)$ (for $T=1$ ). Only the imaginary parts are capable of producing $\zeta(n)$ 's with $n$ odd.

A vacuum diagram in our model involves a multiple integral on the unit circle with an integrand which is a product of propagators (2.1). (One of the integrations is redundant due to translation invariance.) The result of the integrations can obviously be decomposed as a sum of multiple $\zeta$ values. Our model thus defines a map from the set of weighted vacuum graphs to the (vector space generated by) the Euler-Zagier sums. This map is, moreover, easily seen to be surjective; it will be immediately convincing that with the above Feynman rules, the sea shell diagram (Figure 2.1) evaluates to

$$
\lambda^{\sum k_{i}}(g \bar{g})^{2 m-1} \zeta\left(k_{1}, \ldots, k_{m}\right) .
$$

The $u$-integrations produce $\delta$ functions for momentum conservation at every vertex, while the Fourier sums of the inserted $g^{(0)}$ propagators yield Heaviside step functions leading to the desired ordering of the remaining sums.

3. The elementary vertex integrals. Now we start on an investigation of the properties of the Feynman integrals in $x(=u)$-space. An obvious first step is to consider 


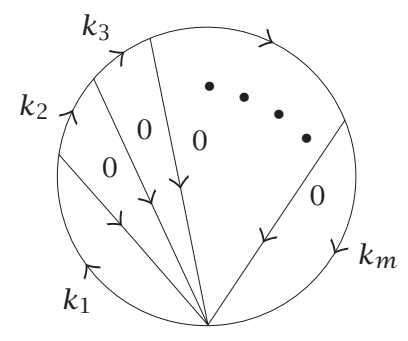

FiguRE 2.1. Sea shell diagram representing the general Euler-Zagier sum.

the folding of the elementary vertices with arbitrary sets of propagators. We denote the elementary vertex integral by

$$
\begin{aligned}
I_{k_{1} \cdots k_{p}}^{l_{1} \cdots l_{q}}\left(u_{1}, \ldots, u_{p+q}\right) \equiv & \int_{0}^{1} d u g^{\left(k_{1}\right)}\left(u_{1}-u\right) \cdots g^{\left(k_{p}\right)}\left(u_{p}-u\right) \\
& \times g^{\left(l_{1}\right)}\left(u-u_{p+1}\right) \cdots g^{\left(l_{q}\right)}\left(u-u_{p+q}\right) .
\end{aligned}
$$

We note that it has the following obvious properties:

$$
\begin{aligned}
& I_{k_{1} \cdots k_{p}}^{l_{1} \cdots l_{q}}=0 \quad \text { if } p=0 \text { or } q=0, \\
& \bar{I}_{k_{1} \cdots k_{p}}^{l_{1} \cdots l_{q}}=(-1)^{\sum_{i=1}^{p} k_{i}+\sum_{j=1}^{q} l_{j}} I_{l_{1} \cdots l_{q}}^{k_{1} \cdots k_{p}} .
\end{aligned}
$$

3.1. Two-vertex integral. By construction two-point vertices can be integrated out trivially,

$$
\int_{0}^{1} d u_{3} g_{13}^{(k)} g_{32}^{(l)}=g_{12}^{(k+l)}
$$

3.2. Three-vertex integral. The evaluation of vertex integrals is complicated by singularities which can appear due to the presence of the cotangent function in $g^{(0)}$. Integrals involving $\cot \left(\pi u_{12}\right)$ need to be performed using the principal value prescription. One way of calculating them is to transform them into complex contour integrals via the substitution $z=\exp (2 \pi i u)$,

$$
\int_{0}^{1} d u \prod_{k=1}^{n} \cot \left(\pi\left(u-u_{k}\right)\right)=i^{n} \oint \frac{d z}{2 \pi i z} \prod_{k=1}^{n} \frac{z+z_{k}}{z-z_{k}}
$$

Those are evaluated by means of residues (the poles on the contour give half values due to the principal value prescription). (We remark that, if one expresses the propagators of the sea shell diagram via (2.2) and (2.7), and then calculates the $u_{i^{-}}$ integrals by means of residues, then one arrives precisely at Kontsevich's integral representation [26] for the multiple $\zeta$ sum.) The first few integrals are 


$$
\begin{aligned}
\int_{0}^{1} d u \cot \left(\pi\left(u-u_{1}\right)\right)= & 0 \\
\int_{0}^{1} d u \prod_{k=1}^{2} \cot \left(\pi\left(u-u_{k}\right)\right)= & \delta\left(u_{1}-u_{2}\right)-1 \\
\int_{0}^{1} d u \prod_{k=1}^{3} \cot \left(\pi\left(u-u_{k}\right)\right)= & \delta\left(u_{1}-u_{2}\right) \cot \left(\pi\left(u_{2}-u_{3}\right)\right) \\
& +\delta\left(u_{1}-u_{3}\right) \cot \left(\pi\left(u_{1}-u_{2}\right)\right) \\
& +\delta\left(u_{2}-u_{3}\right) \cot \left(\pi\left(u_{2}-u_{1}\right)\right) .
\end{aligned}
$$

Alternatively one can also calculate those integrals recursively using, under the integral, the following identity:

$$
\begin{aligned}
& \cot \left(\pi u_{12}\right) \cot \left(\pi u_{13}\right)+\cot \left(\pi u_{21}\right) \cot \left(\pi u_{23}\right)+\cot \left(\pi u_{31}\right) \cot \left(\pi u_{32}\right) \\
& \quad=-1+\delta\left(u_{12}\right) \delta\left(u_{13}\right) .
\end{aligned}
$$

This identity will be of further use later on. With these results, the vertex integral of three propagators $g^{(0)}$ is

$$
\begin{aligned}
I_{00}^{0}\left(u_{1}, u_{2}, u_{3}\right)= & \int_{0}^{1} d u g^{(0)}\left(u_{1}-u\right) g^{(0)}\left(u_{2}-u\right) g^{(0)}\left(u-u_{3}\right) \\
=\frac{1}{4}[ & \delta\left(u_{13}\right) \delta\left(u_{23}\right)-\delta\left(u_{13}\right)-\delta\left(u_{23}\right)+1+\left(\delta\left(u_{13}\right)-1\right) i \cot \left(\pi u_{23}\right) \\
& \left.\quad+\left(\delta\left(u_{23}\right)-1\right) i \cot \left(\pi u_{13}\right)-\cot \left(\pi u_{23}\right) \cot \left(\pi u_{13}\right)\right],
\end{aligned}
$$

and can be identified as

$$
I_{00}^{0}\left(u_{1}, u_{2}, u_{3}\right)=g_{13}^{(0)} g_{23}^{(0)} .
$$

Applying the identity (3.6) gives also

$$
\begin{aligned}
I_{00}^{0}\left(u_{1}, u_{2}, u_{3}\right) & =g_{12}^{(0)} g_{23}^{(0)}-g_{12}^{(0)} g_{13}^{(0)}-\frac{1}{2} g_{13}^{(0)} \\
& =g_{21}^{(0)} g_{13}^{(0)}-g_{21}^{(0)} g_{23}^{(0)}-\frac{1}{2} g_{23}^{(0)} \\
& =g_{12}^{(0)} g_{23}^{(0)}+g_{21}^{(0)} g_{13}^{(0)}-\frac{1}{2} \delta_{12} g_{13}^{(0)} .
\end{aligned}
$$

Folding of (3.8) with $\left\langle u_{1^{\prime}}\left|\partial^{-n}\right| u_{1}\right\rangle$ and $\left\langle u_{2^{\prime}}\left|\partial^{-m}\right| u_{2}\right\rangle$ leads to

$$
\begin{aligned}
I_{n m}^{0}\left(u_{1}, u_{2}, u_{3}\right) & =\int_{0}^{1} d u g^{(n)}\left(u_{1}-u\right) g^{(m)}\left(u_{2}-u\right) g^{(0)}\left(u-u_{3}\right) \\
& =g_{13}^{(n)} g_{23}^{(m)},
\end{aligned}
$$

whereas the forms (3.9) can be folded with $\left\langle u_{3}\left|\partial^{-k}\right| u_{3^{\prime}}\right\rangle$

$$
\begin{aligned}
I_{00}^{k}\left(u_{1}, u_{2}, u_{3}\right) & =\int_{0}^{1} d u g^{(0)}\left(u_{1}-u\right) g^{(0)}\left(u_{2}-u\right) g^{(k)}\left(u-u_{3}\right) \\
& =g_{12}^{(0)} g_{23}^{(k)}-g_{12}^{(0)} g_{13}^{(k)}-\frac{1}{2} g_{13}^{(k)} \\
& =g_{21}^{(0)} g_{13}^{(k)}-g_{21}^{(0)} g_{23}^{(k)}-\frac{1}{2} g_{23}^{(k)} \\
& =g_{12}^{(0)} g_{23}^{(k)}+g_{21}^{(0)} g_{13}^{(k)}-\frac{1}{2} \delta_{12} g_{13}^{(k)} .
\end{aligned}
$$




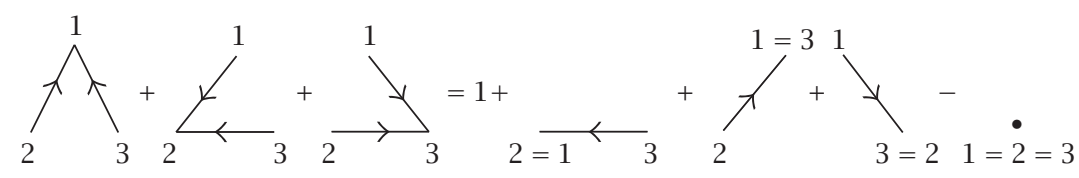

FIGURE 3.1. Three-point relation.

Nontrivial is the case

$$
\begin{aligned}
I_{0 k}^{l}\left(u_{1}, u_{2}, u_{3}\right) & =\int_{0}^{1} d u g^{(0)}\left(u-u_{1}\right) g^{(k)}\left(u-u_{2}\right) g^{(l)}\left(u_{3}-u\right) \\
& =\operatorname{Li}_{l k}\left(z_{31}, z_{12}\right)
\end{aligned}
$$

The general case of all three indices different from zero can be reduced to this case by partial integrations, for example,

$$
\begin{aligned}
I_{11}^{1}\left(u_{1}, u_{2}, u_{3}\right)= & \int_{0}^{1} d u g^{(1)}\left(u_{41}\right) g^{(1)}\left(u_{42}\right) g^{(1)}\left(u_{34}\right) \\
= & \int_{0}^{1} d u g^{(1)}\left(u_{41}\right) g^{(0)}\left(u_{42}\right) g^{(2)}\left(u_{34}\right) \\
& +\int_{0}^{1} d u g^{(0)}\left(u_{41}\right) g^{(1)}\left(u_{42}\right) g^{(2)}\left(u_{34}\right) \\
= & \operatorname{Li}_{21}\left(z_{31}, z_{12}\right)+\operatorname{Li}_{21}\left(z_{32}, z_{21}\right)
\end{aligned}
$$

3.3. A three-point relation. Beyond the three-point case the systematic investigation of the elementary vertex integrals becomes cumbersome. We will be satisfied here to note that, from the representation (2.8) for $g^{(0)}$ and the identity (3.6) we easily derive the following pair of (complex conjugate) three-point identities:

$$
\begin{aligned}
& g_{21}^{(0)} g_{31}^{(0)}+g_{12}^{(0)} g_{32}^{(0)}+g_{13}^{(0)} g_{23}^{(0)}=1+\delta_{12} g_{32}^{(0)}+\delta_{31} g_{21}^{(0)}+\delta_{23} g_{13}^{(0)}-\delta_{12} \delta_{13} \\
& g_{12}^{(0)} g_{13}^{(0)}+g_{21}^{(0)} g_{23}^{(0)}+g_{31}^{(0)} g_{32}^{(0)}=1+\delta_{12} g_{23}^{(0)}+\delta_{31} g_{12}^{(0)}+\delta_{23} g_{31}^{(0)}-\delta_{12} \delta_{13}
\end{aligned}
$$

We may represent the first identity graphically as in Figure 3.1. The second identity has all arrows reversed. Those identities can be used to transform any vertex integral involving two $g^{(0)}$ 's which are either both ingoing or both outgoing.

By iteration of this three-point identity we can contruct an analogous identity for an arbitrary number of points. The formulas are rather cumbersome and are not given here.

4. Derivation of multiple $\zeta$ relations. Now we show how to use the formalism developed above for deriving a large class of multiple $\zeta$ relations by simple manipulations on graphs, with no need to ever explicitly write down sums. In those manipulations we will make use of the following elements:

(1) the triviality of the real part of $g^{(0)}, \bar{g}_{12}^{(0)}=(1 / 2)\left(\delta_{12}-1\right)$ (see (2.8));

(2) partial integrations;

(3) the three-point relations (3.14); 
(4) the vanishing of diagrams containing a vertex with only ingoing or only outgoing propagators;

(5) the two-vertex integration formula (3.3).

At intermediate steps sometimes ill-defined sums will appear such as $\zeta(1)$. Those will be always cancelled out in the final results. The appearance of divergent sums could be easily avoided using a regularization such as in [9] but we will not do so here.

4.1. Length two. We begin at length two. The simplest way of arriving at identities is provided by the first one of the points listed above. Consider the sea shell diagram at length two (Figure 4.1a), representing $\zeta(a, b)$, and the same diagram with the middle propagator reversed (Figure 4.1b), representing $\zeta(b, a)$. (We will generally disregard the coupling constant factors in the following.) Adding up both diagrams we can replace the middle propagator by twice its real part (Figure 4.1). Since $\overline{\mathfrak{g}}_{12}^{(0)}=(1 / 2)\left(\delta_{12}-1\right)$, this diagram can then be replaced by the sum of the two diagrams shown in the right-hand side of Figure 4.1. Using (3.3) on the rightmost one, we obtain the identity

$$
\zeta(a, b)+\zeta(b, a)=\zeta(a) \zeta(b)-\zeta(a+b) .
$$

This identity is well known [9, 21], and has been named reflection formula in [9].

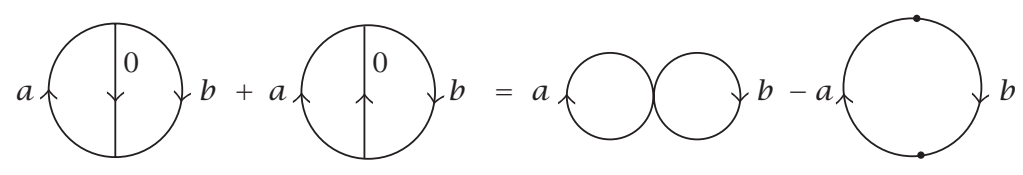

(a)

(b)

FIGURE 4.1. Diagrammatic representation of the reflection identity.

Another way of obtaining identities is partial integration. Instead of considering the sea shell diagram (Figure 4.1a), which represents $\zeta(a, b)$, consider the more general diagram (Figure 4.2) that represents a number $G_{a, b, c}=G_{a, c, b}$. For $a=0, b=0$, or $c=0$, it can be represented by multiple $\zeta$ functions,

$$
G_{0, b, c}=\zeta(b) \zeta(c), \quad G_{a, b, 0}=G_{a, 0, b}=\zeta(a, b) .
$$

To see the first of these identities, add the same diagram with the left propagator reversed, and use elements one and four from the above list. For the later discussion we

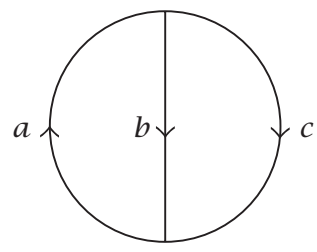

FIGURE 4.2. Auxiliary diagrams for multiple $\zeta$ relations of length two. 


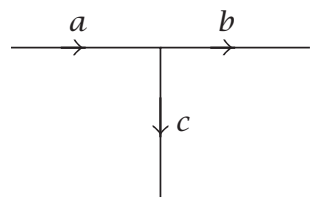

(a)

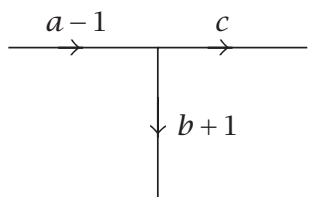

(b)

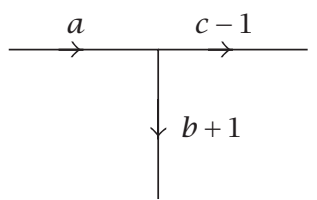

(c)

FIGURE 4.3. Integration by parts.

refer to these diagrams as zeta diagrams in contrast to the other, non-zeta diagrams. If both $a$ and $c$ are greater than 1, we use integration by parts at the upper vertex according to Figure 4.3 and obtain

$$
G_{a, b, c}=G_{a-1, b+1, c}-G_{a, b+1, c-1} .
$$

This can be repeated until either $a=0$ or $c=0$

$$
\begin{aligned}
G_{a, b, c}= & \sum_{n=1}^{c}(-1)^{c+n}\left(\begin{array}{c}
a+c-n-1 \\
a-1
\end{array}\right) G_{0, a+b+c-n, n} \\
& +\sum_{n=1}^{a}(-1)^{c}\left(\begin{array}{c}
a+c-n-1 \\
c-1
\end{array}\right) G_{n, a+b+c-n, 0} .
\end{aligned}
$$

The right-hand side of this equation can be translated immediately into $\zeta$ values by (4.2). For $b=0$, we obtain the relation

$$
\begin{gathered}
\zeta(a, b)=(-1)^{b}\left[\sum_{n=1}^{b}(-1)^{n}\left(\begin{array}{c}
a+b-n-1 \\
a-1
\end{array}\right) \zeta(n) \zeta(a+b-n)\right. \\
\left.+\sum_{n=1}^{a}\left(\begin{array}{c}
a+b-n-1 \\
b-1
\end{array}\right) \zeta(n, a+b-n)\right] .
\end{gathered}
$$

The divergent $\zeta(1)$ and $\zeta(1, a)$ appear here always in the combination

$$
\zeta(1) \zeta(a+b-1)-\zeta(1, a+b-1)
$$

which can be reexpressed in terms of convergent sums by the reflection identity (4.1). In this way, we obtain

$$
\begin{aligned}
\zeta(a, b)=(-1)^{b} & {\left[\sum_{n=2}^{b}(-1)^{n}\left(\begin{array}{c}
a+b-n-1 \\
a-1
\end{array}\right) \zeta(n) \zeta(a+b-n)\right.} \\
& +\sum_{n=2}^{a}\left(\begin{array}{c}
a+b-n-1 \\
b-1
\end{array}\right) \zeta(n, a+b-n) \\
& \left.-\left(\begin{array}{c}
a+b-2 \\
a-1
\end{array}\right)(\zeta(a+b)+\zeta(a+b-1,1))\right]
\end{aligned}
$$

Here on the left-hand side we must assume that $a>1$ if regularization is to be avoided.

4.2. Length three. Proceeding to sums of length three, again begin by exploiting the triviality of $\overline{\boldsymbol{g}}^{(0)}$. Since there are two $g^{(0)}$ propagators, we have now several possi- 


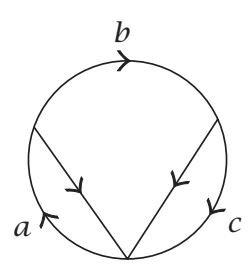

(a)

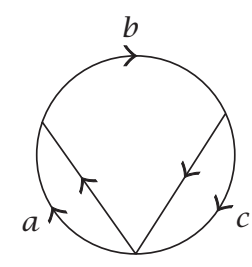

(b)

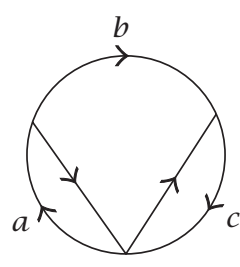

(c)

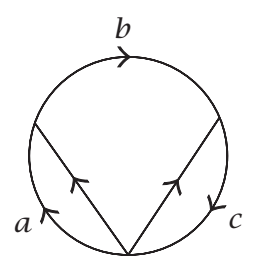

(d)

FIGURE 4.4. Diagrams related to $\zeta(a, b, c)$. The propagators without label are $g^{(0)}$ propagetors.

bilities. Figure 4.4 shows the "standard" sea shell diagram, representing $\zeta(a, b, c)$, as well as the diagrams related to it by a change of direction of one or both of the $g^{(0)}$ propagators. Those diagrams represent the quantities

(a) $\zeta(a, b, c)$,

(b) $\zeta(b, a, c)+\zeta(b, c, a)+\zeta(b, a+c)$,

(c) $\zeta(a, c, b)+\zeta(c, a, b)+\zeta(a+c, b)$,

(d) $\zeta(c, b, a)$.

Adding those diagrams in pairs to create $\overline{\mathfrak{g}}^{(0)}$ 's one obtains the following four identities,

$$
\begin{aligned}
& \zeta(a, b, c)+\zeta(b, a, c)+\zeta(b, c, a)+\zeta(b, a+c)+\zeta(a+b, c)-\zeta(a) \zeta(b, c)=0 \\
& \zeta(a, b, c)+\zeta(a, c, b)+\zeta(c, a, b)+\zeta(a+c, b)+\zeta(a, b+c)-\zeta(c) \zeta(a, b)=0 \\
& \zeta(b, a, c)+\zeta(b, c, a)+\zeta(c, b, a)+\zeta(b, a+c)+\zeta(b+c, a)-\zeta(c) \zeta(b, a)=0 \\
& \zeta(a, c, b)+\zeta(c, a, b)+\zeta(c, b, a)+\zeta(a+c, b)+\zeta(c, a+b)-\zeta(a) \zeta(c, b)=0 .
\end{aligned}
$$

These identities generalise the reflection identity (4.5). We call them permutation identities.

At length three, we can also make use of the three-point identities (3.14). We consider again Figure 4.4a, and apply the first one of the three-point relations to the two $g^{(0)}$ propagators running into the root vertex. The result is the diagrammatic identity shown in Figure 4.5. It can be translated, term by term, into the following $\zeta$ identity:

$$
\begin{aligned}
\zeta(a, b, c)= & -\zeta(b, c, a)-\zeta(c, a, b)+\zeta(a+b+c)+\zeta(a) \zeta(b, c) \\
& +\zeta(b) \zeta(c, a)+\zeta(c) \zeta(a, b)-\zeta(a) \zeta(b) \zeta(c) .
\end{aligned}
$$

The identities (4.9) through (4.11) can be obtained by applying the permutations $b \rightarrow a \rightarrow c \rightarrow b, a \leftrightarrow c, b \leftrightarrow c$ on (4.8). Taking the latter identity and applying all permutations, we obtain six identities which can be written as

$$
M \vec{z}=\vec{a},
$$

where $\vec{z}=(\zeta(a, b, c), \zeta(a, c, b), \ldots, \zeta(c, b, a))$ (all permutations of the arguments) and $\vec{a}$ is a vector which contains only $\zeta$ values of length one and two. The rank of the coefficient matrix $M$ is 4 , that is, four zeta values in $\vec{z}$ can be expressed by the other two 


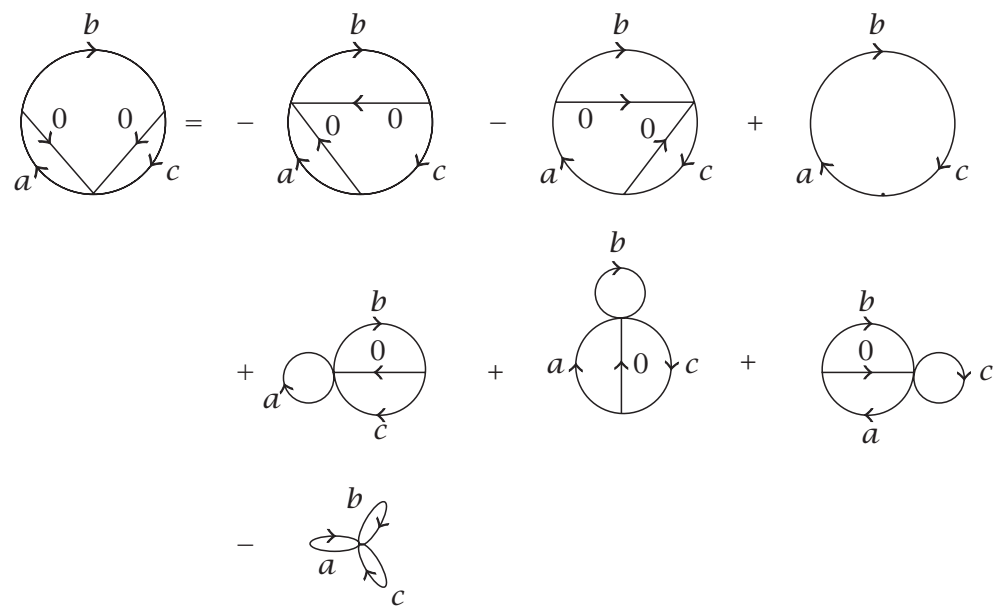

FIGURE 4.5. Diagrammatic identity derived from the three-point identity.

(and lower-length zeta values), for example, by $\zeta(a, b, c)$ and $\zeta(a, c, b)$. Taking into account also relation (4.12) the coefficient matrix $M$ gets more rows but the rank does not change. Therefore, this relation is up to lower-length identities not independent of (4.8). When two of the arguments $a, b, c$ coincide, say $b=c, \vec{z}$ becomes $(\zeta(a, b, b)$, $\zeta(b, a, b), \zeta(b, b, a))$ and the rank of $M$ reduces to 2. For all arguments coinciding, the rank of $M$ is 1 .

There is another possibility to derive an identity from Figure $4.4 \mathrm{~d}$ which generalises immediately to $\zeta$ values of larger length. Every $\boldsymbol{g}^{(0)}$ propagator in Figure $4.4 \mathrm{~d}$ can be replaced by the reverted propagator according to

$$
\mathfrak{g}_{12}^{(0)}=-\boldsymbol{g}_{21}^{(0)}+\delta_{12}-1 .
$$

This leads to a sum of diagrams where all occurring $g^{(0)}$ propagators are directed towards the root vertex. The resulting identity

$$
\begin{aligned}
\zeta(c, b, a)= & \zeta(a, b, c)-\zeta(a, b) \zeta(c)+\zeta(a, b+c) \\
& +\zeta(a)(-\zeta(b, c)+\zeta(b) \zeta(c)-\zeta(b+c)) \\
& +\zeta(a+b, c)-\zeta(a+b) \zeta(c)+\zeta(a+b+c)
\end{aligned}
$$

can be obtained also by subtracting (4.8) from (4.11) and applying appropriately the length-two identity (4.1), thus it is not a new identity.

Partial integrations yield additional identities. As for $\zeta$ values of length two, we consider the more general figure (Figure 4.6) which evaluate to the numbers $G_{a, k, b, l, c}=$ $G_{a, k, b, c, l}$. Some of these numbers can be identified with $\zeta$ values:

$$
G_{a, 0, b, 0, c}=G_{a, 0, b, c, 0}=\zeta(a, b, c), \quad G_{0, k, b, 0, c}=G_{0, k, b, c, 0}=\zeta(k) \zeta(b, c) .
$$

We start with $\zeta(a, b, c)=G_{a, 0, b, 0, c}$. First, we integrate by parts at the upper-right vertex until $b=0$ or $c=0$. The combinatorics is the same as in (4.4). The terms with $c=0$ can 


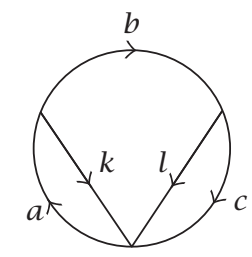

FIGURE 4.6. Auxiliary diagrams for partial integrations at length three.

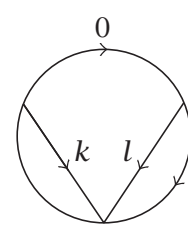

(a)

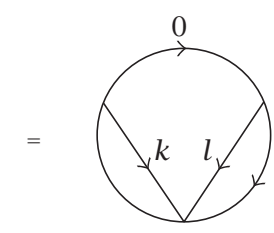

(b)

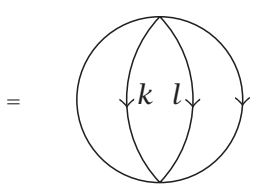

(d)

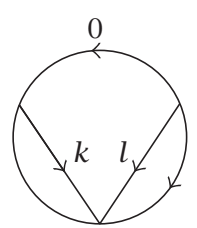

(c)

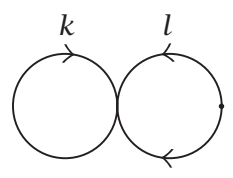

(e)

FIGURE 4.7. Identity which proves that $k$ and $l$ can be exchanged when the upper propagator is zero. Diagram (c) is zero because no propagator goes into the upper right vertex. Diagram (e) is zero for the same reason at the right vertex.

be identified with $\zeta$ values. In the terms where $b=0$, the two inner propagators can be exchanged according to the identity depicted in Figure 4.7. After this exchange, we can integrate by parts at the upper-left vertex until terms with $a=0$ or $k=0$ arise. In terms of $\zeta$ values, we obtain

$$
\begin{aligned}
\zeta(a, b, c)=(-1)^{b} \sum_{n=1}^{c} & {\left[\sum_{m=1}^{a}\left(\begin{array}{c}
a+b+c-m-n-1 \\
b-1, a-m, c-n
\end{array}\right) \zeta(m, a+b+c-m-n, n)\right.} \\
& +\sum_{m=1}^{b+c-n}(-1)^{m}\left(\begin{array}{c}
b+c-n-1 \\
b-1
\end{array}\right)\left(\begin{array}{c}
a+b+c-m-n-1 \\
a-1
\end{array}\right) \\
& \times \zeta(m) \zeta(a+b+c-m-n, n)] \\
+ & \sum_{n=1}^{b}(-1)^{c}\left(\begin{array}{c}
b+c-n-1 \\
c-1
\end{array}\right) \zeta(a, n, b+c-n),
\end{aligned}
$$

where on the left-hand side we have again to require that $a>1$. The divergent terms 
for $m=1$ appear always in a combination which can be eliminated by identity (4.9),

$$
\zeta(1) \zeta(a, b)-\zeta(1, a, b)=\zeta(a, b, 1)+\zeta(a, 1, b)+\zeta(a+1, b)+\zeta(a, b+1) .
$$

Alternatively we may after the first step, in the terms with $b=0$, rather than interchanging the two inner propagators, those labelled " $k$ " and " $l$ " in Figure 4.6, instead interchange propagators " $k$ " and " $c$." Proceeding in the same way as before we obtain the following identity:

$$
\begin{aligned}
\zeta(a, b, c)= & (-1)^{c} \sum_{n=1}^{b}\left(\begin{array}{c}
b+c-n-1 \\
c-1
\end{array}\right) \zeta(a, n, b+c-n) \\
& +(-1)^{c} \sum_{n=1}^{c} \sum_{m=1}^{a}\left(\begin{array}{c}
b+c-n-1 \\
b-1
\end{array}\right)\left(\begin{array}{c}
a-m+n-1 \\
n-1
\end{array}\right) \\
& \times \zeta(m, a-m+n, b-n+c) \\
& +\sum_{n=1}^{c} \sum_{m=1}^{n}(-1)^{c-m}\left(\begin{array}{c}
a-m+n-1 \\
a-1
\end{array}\right)\left(\begin{array}{c}
b-n+c-1 \\
b-1
\end{array}\right) \\
& \times \zeta(m) \zeta(a-m+n, b-n+c) .
\end{aligned}
$$

Here again the terms involving a $\zeta(1, \ldots)$ appear only in the combination (4.9) and thus can be removed without the need for regularization.

4.3. Arbitrary length. All three different procedures which we have used for constructing multiple $\zeta$ identities-partial integrations, reversion of $g^{(0)}$ propagators, and the use of the three-point identity-can be generalised to the arbitrary length case without difficulty.

The generalisation of the permutation identities to an arbitrary length is based on finding representations of sea shell diagrams with reverted inner $\boldsymbol{g}^{(0)}$ propagators, which are added in order to use the two-point relation (4.14). Consider first the simplest case with one reverted propagator Figure 4.8. Figures $4.8 \mathrm{~b}, 4.8 \mathrm{c}$, and $4.8 \mathrm{~d}$ can be expressed immediately by $\zeta$ values. Figures $4.8 \mathrm{a}, 4.8 \mathrm{~b}$, and $4.8 \mathrm{c}$ represent the series

$$
\sum_{\substack{n_{1}, \ldots, n_{l} \\ n_{1}>\cdots>n_{m}>0, n_{m+1}>\cdots>n_{l}>0}}^{\infty} \frac{1}{n_{1}^{k_{1}} n_{2}^{k_{2}} \cdots n_{l}^{k_{l}}} .
$$

By decomposing the summation range into regions where the $n_{\lambda}$ 's are completely ordered, we obtain a sum of $\zeta$ values which can be constructed as follows.

Denote by $M_{a_{1}, a_{2}, a_{12}} \subset\{\{1\},\{2\},\{1,2\}\} a_{1}+a_{2}+a_{12}$ the set of all $\left(a_{1}+a_{2}+a_{12}\right)$-tuples consisting of $a_{1}$ elements $\{1\}, a_{2}$ elements $\{2\}$ and $a_{12}$ elements $\{1,2\}$. Further, we define a map $\left(l \equiv m+m^{\prime}\right)$

$$
\begin{gathered}
\rho_{m, m^{\prime}}^{a}: \mathbb{N}^{l} \times M_{m-a, m^{\prime}-a, a} \longrightarrow \mathbb{N}^{l-a}, \\
\left(k_{1}, \ldots, k_{m} ; k_{m+1}, \ldots, k_{l} ; m_{1}, \ldots, m_{l-a}\right) \longmapsto\left(b_{1}+b_{1}^{\prime}, \ldots, b_{l-a}+b_{l-a}^{\prime}\right),
\end{gathered}
$$




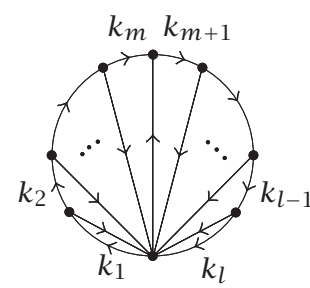

(a)

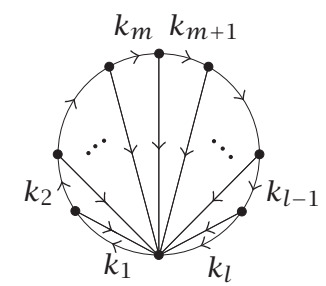

(b)

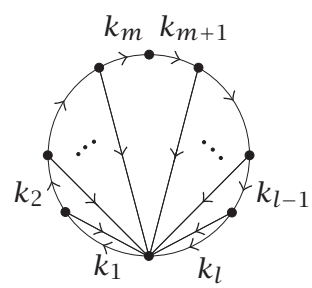

(c)

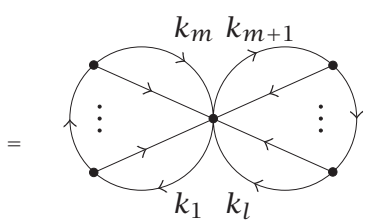

(d)

FIGURE 4.8. Diagrammatic representation of permutation identities for arbitrary length. The propagators without label are $g^{(0)}$ propagators.

where $\left(b_{1}, \ldots, b_{l-a}\right)$ results from $\left(m_{1}, \ldots, m_{l-a}\right)$ by replacing $\{2\}$ with 0 , and $\{1\}$ and $\{1,2\}$ with $k_{1}, \ldots, k_{m}$ (in this order); $\left(b_{1}^{\prime}, \ldots, b_{l-a}^{\prime}\right)$ results from $\left(m_{1}, \ldots, m_{l-a}\right)$ by replacing $\{1\}$ with 0 , and $\{2\}$ and $\{1,2\}$ with $k_{m+1}, \ldots, k_{l}$ (in this order). For example,

$$
\rho_{2,2}^{1}\left(k_{1}, k_{2} ; k_{3}, k_{4} ;\{1\},\{1,2\},\{2\}\right)=\left(k_{1}, k_{2}+k_{3}, k_{4}\right) .
$$

With these definitions, the identity depicted in Figure 4.8 can be written as

$$
\begin{aligned}
& \sum_{a=0}^{\min (m, l-m)} \sum_{\xi \in M_{m-a, l-m-a, a}} \zeta\left(\rho_{m, l-m}^{a}\left(k_{1}, \ldots, k_{m} ; k_{m+1}, \ldots, k_{l} ; \xi\right)\right) \\
& \quad=\zeta\left(k_{1}, \ldots, k_{m}\right) \zeta\left(k_{m+1}, \ldots, k_{l}\right) .
\end{aligned}
$$

The generalisation to the cases with more than one reverted propagator should be obvious. But we note that in these cases the $\zeta$ values of maximal length appear in combinations which can be constructed also from the identities (4.23). Thus, we conjecture that all identities which are based on the reordering of summation ranges are generated by (4.23).

Imitating the considerations in (4.13), we can write all permutation identities (4.23) for fixed $l$ (but varying $m$ ), where the arguments of the length-one $\zeta$ values are taken from the set $\left\{k_{1}, \ldots, k_{l}\right\}$ in all possible orderings, in the form (4.13), where now the components of $\vec{z}$ consist of all different $\zeta$ values which result from $\zeta\left(k_{1}, \ldots, k_{l}\right)$ by permutations of the arguments; $\vec{a}$ contains only $\zeta$ values of length less than 1 . Assuming $k_{1}, \ldots, k_{l}$ mutually different, we found that the coefficient matrix $M$ has rank 18 for $l=4$ and rank 96 for $l=5$. These results suggest that for length one the rank is $l$ ! $-(l-1)$ ! and that the permutation identities suffice to express the $l$ ! considered $\zeta$ values of length one by the subset of $(l-1)$ ! $\zeta$ values where one of the arguments is held fixed at a certain position. 
To generalise the partial integration procedure from length three to length $m$, we can proceed in various ways. For example, we can simply iterate the above exchange of inner propagators. In the first step we apply the same partial integration as in the length-three case to the rightmost vertex of the sea shell diagram (Figure 2.1). For those terms in the result where $k_{m-1}=0$ we make use of this new $g^{(0)}$ propagator to interchange the adjacent inner propagators. Then we perform partial integrations on the next-to-rightmost vertex until either $k_{m-2}=0$ or the next inner propagator becomes the zero propagator. In the first case the procedure continues with another interchange of inner propagators. After maximally $m-1$ such propagator interchanges, proceeding from right to left, the final step is reached, which is again the same as in the length-three case.

In the first step we have the same ambiguity as before. Depending on its resolution, we arrive at a generalisation of either (4.18) or (4.19). We give here the formula generalising (4.19),

$$
\begin{aligned}
& \zeta\left(k_{1}, \ldots, k_{m}\right) \\
& =(-1)^{k_{m}} \sum_{n_{m-1}=1}^{k_{m-1}}\left(\begin{array}{c}
k_{m-1}-n_{m-1}+n_{m}-1 \\
k_{m}-1
\end{array}\right) \zeta\left(k_{1}, \ldots, k_{m-2}, n_{m-1}, k_{m-1}-n_{m-1}+n_{m}\right) \\
& +(-1)^{k_{m}} \sum_{n_{m-1}=1}^{n_{m}} \sum_{n_{m-2}=1}^{k_{m-2}}\left(\begin{array}{c}
k_{m-1}-n_{m-1}+n_{m}-1 \\
k_{m-1}-1
\end{array}\right)\left(\begin{array}{c}
k_{m-2}-n_{m-2}+n_{m-1}-1 \\
n_{m-1}-1
\end{array}\right) \\
& \times \zeta\left(k_{1}, \ldots, k_{m-3}, n_{m-2}, k_{m-2}-n_{m-2}+n_{m-1}, k_{m-1}-n_{m-1}+n_{m}\right) \\
& +(-1)^{k_{m}} \sum_{n_{m-1}=1}^{n_{m}} \sum_{n_{m-2}=1}^{n_{m-1}} \sum_{n_{m-3}=1}^{k_{m-3}}\left(\begin{array}{c}
k_{m-1}-n_{m-1}+n_{m}-1 \\
k_{m-1}-1
\end{array}\right)\left(\begin{array}{c}
k_{m-2}-n_{m-2}+n_{m-1}-1 \\
k_{m-2}-1
\end{array}\right) \\
& \times\left(\begin{array}{c}
k_{m-3}-n_{m-3}+n_{m-2}-1 \\
n_{m-2}-1
\end{array}\right) \zeta\left(k_{1}, \ldots, k_{m-4}, n_{m-3}, k_{m-3}-n_{m-3}+n_{m-2}, \ldots\right) \\
& \vdots \\
& +(-1)^{k_{m}} \sum_{n_{m-1}=1}^{n_{m}} \sum_{n_{m-2}=1}^{n_{m-1}} \cdots \sum_{n_{2}=1}^{n_{3}} \sum_{n_{1}=1}^{k_{1}} \prod_{n=2}^{m-1}\left(\begin{array}{c}
k_{n}-n_{n}+n_{n+1}-1 \\
k_{n}-1
\end{array}\right)\left(\begin{array}{c}
k_{1}-n_{1}+n_{2}-1 \\
n_{2}-1
\end{array}\right) \\
& \times \zeta\left(n_{1}, k_{1}-n_{1}+n_{2}, \ldots, k_{m-1}-n_{m-1}+n_{m}\right) \\
& +\sum_{n_{m-1}=1}^{n_{m}} \sum_{n_{m-2}=1}^{n_{m-1}} \cdots \sum_{n_{1}=1}^{n_{2}}(-1)^{k_{m}-n_{1}} \prod_{n=1}^{m-1}\left(\begin{array}{c}
k_{n}-n_{n}+n_{n+1}-1 \\
k_{n}-1
\end{array}\right) \\
& \times \zeta\left(n_{1}\right) \zeta\left(k_{1}-n_{1}+n_{2}, k_{2}-n_{2}+n_{3}, \ldots, k_{m-1}-n_{m-1}+n_{m}\right),
\end{aligned}
$$

where $n_{m} \equiv k_{m}$. We also give the special case $k_{m}=1$ of this formula which is particularly simple,

$$
\begin{aligned}
\zeta\left(k_{1}, \ldots, k_{m-1}, 1\right)= & \zeta(1) \zeta\left(k_{1}, \ldots, k_{m-1}\right) \\
& -\sum_{\kappa=1}^{m-1} \sum_{n_{\kappa}=1}^{k_{\kappa}} \zeta\left(k_{1}, \ldots, k_{\kappa-1}, k_{\kappa}+1-n_{\kappa}, n_{\kappa}, k_{\kappa+1}, \ldots, k_{m-1}\right) .
\end{aligned}
$$


The terms involving $\zeta(1, \ldots)$ can be removed by means of a special case of (4.23), namely

$$
\begin{aligned}
\zeta(1) & \zeta\left(k_{1}, \ldots, k_{m-1}\right)-\zeta\left(1, k_{1}, \ldots, k_{m-1}\right) \\
& =\sum_{\kappa=1}^{m-1}\left[\zeta\left(k_{1}, \ldots, k_{\kappa-1}, k_{\kappa}+1, k_{\kappa+1}, \ldots, k_{m-1}\right)+\zeta\left(k_{1}, \ldots, k_{\kappa}, 1, k_{\kappa+1}, \ldots, k_{m-1}\right)\right] .
\end{aligned}
$$

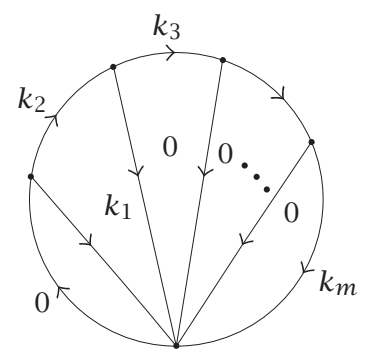

FIGURE 4.9. Modified sea shell diagram.

Up to here, the partial integrations were sequentially applied starting at the rightmost vertex and ending at the leftmost one. An interesting alternative is to do the opposite. Consider Figure 4.9. As in the derivation of the other partial-integration identities, this diagram represents $\zeta\left(k_{1}\right) \zeta\left(k_{2}, \ldots, k_{m}\right)$. On the other hand, we can use partial integrations at the leftmost vertex until we have only terms where either $k_{1}$ or $k_{2}$ became 0 . If $k_{1}=0$ then the resulting diagram represents a multiple $\zeta$ value. If $k_{2}=0$ then we exchange the adjacent inner propagators and repeat the whole procedure at the next-to-leftmost vertex. This continues until the rightmost vertex is reached, where after the partial integrations all terms represent multiple $\zeta$ values. The result is

$$
\begin{aligned}
\zeta\left(k_{1}\right) & \zeta\left(k_{2}, \ldots, k_{m}\right) \\
= & \sum_{n_{1}=1}^{k_{2}}\left(\begin{array}{c}
k_{2}+k_{1}-n_{1}-1 \\
k_{1}-1
\end{array}\right) \zeta\left(k_{2}+k_{1}-n_{1}, n_{1}, k_{3}, \ldots, k_{m}\right) \\
& +\sum_{\kappa=2}^{m-1} \sum_{n_{1}=1}^{n_{0}} \sum_{n_{2}=1}^{n_{1}} \ldots \sum_{n_{\kappa-1}=1}^{n_{\kappa-2}} \sum_{n_{\kappa}=1}^{k_{\kappa+1}} \prod_{\lambda=2}^{\kappa}\left(\begin{array}{c}
k_{\lambda}+n_{\lambda-2}-n_{\lambda-1}-1 \\
k_{\lambda}-1
\end{array}\right)\left(\begin{array}{c}
k_{\kappa+1}+n_{\kappa-1}-n_{\kappa}-1 \\
n_{\kappa-1}-1
\end{array}\right) \\
& \times \zeta\left(k_{2}+n_{0}-n_{1}, k_{3}+n_{1}-n_{2}, \ldots, k_{\kappa+1}+n_{\kappa-1}-n_{\kappa}, n_{\kappa}, k_{\kappa+2}, \ldots, k_{m}\right) \\
& +\sum_{n_{1}=1}^{n_{0}} \sum_{n_{2}=1}^{n_{1}} \ldots \sum_{n_{m-1}=1}^{n_{m-2}} \prod_{\lambda=2}^{m}\left(\begin{array}{c}
k_{\lambda}+n_{\lambda-2}-n_{\lambda-1}-1 \\
k_{\lambda}-1
\end{array}\right) \\
& \times \zeta\left(k_{2}+n_{0}-n_{1}, k_{3}+n_{1}-n_{2}, \ldots, k_{m}+n_{m-2}-n_{m-1}, n_{m-1}\right),
\end{aligned}
$$

$\left(n_{0} \equiv k_{1}\right)$. The right-hand side contains no divergent terms when $k_{1}, k_{2} \geq 2$. For $k_{1}=1$, $k_{2} \geq 2$ it becomes finite when combined with (4.26). 
From our derivation clearly one would expect (4.24) and (4.27) to be equivalent. And indeed, it is a matter of pure combinatorics to show that (4.24) becomes trivially fulfilled if (4.27) is used on the right-hand side. Nevertheless, we suspect that the form (4.27) may be more useful for the application of these formulas to the problem of constructing a minimal basis of independent multiple $\zeta$ sums.

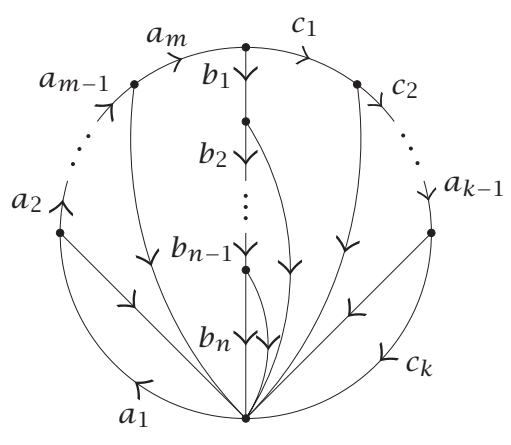

FIGURE 4.10. Peacock diagram. The propagators without label are $g^{(0)}$ propagators.

Equation (4.27) is a special case of a class of identities derived from (weight-length) shuffle algebras. In order to derive that whole class, consider Figure 4.10. It evaluates to a number which we denote by

$$
Z\left(a_{1}, \ldots, a_{m}\left|b_{1}, \ldots, b_{n}\right| c_{1}, \ldots, c_{k}\right)=Z\left(a_{1}, \ldots, a_{m}\left|c_{1}, \ldots, c_{k}\right| b_{1}, \ldots, b_{n}\right) .
$$

Partial integrations at the top vertex yield, similarly to (4.4),

$$
\begin{aligned}
Z(\ldots, & \left.a_{m}, 0\left|b_{1}, b_{2}, \ldots\right| c_{1}, c_{2}, \ldots\right) \\
= & \sum_{v=1}^{b_{1}}\left(\begin{array}{c}
b_{1}+c_{1}-v-1 \\
c_{1}-1
\end{array}\right) Z\left(\ldots, a_{m}, b_{1}+c_{1}-v\left|v, b_{2}, \ldots\right| 0, c_{2}, \ldots\right) \\
& +\sum_{v=1}^{c_{1}}\left(\begin{array}{c}
b_{1}+c_{1}-v-1 \\
b_{1}-1
\end{array}\right) Z\left(\ldots, a_{m}, b_{1}+c_{1}-v\left|0, b_{2}, \ldots\right| v, c_{2}, \ldots\right) .
\end{aligned}
$$

Considerations like in Figure 4.7 show

$$
\begin{aligned}
Z\left(a_{1}, \ldots, a_{m}\left|0, b_{1}, \ldots\right| c_{1}, \ldots\right) & =Z\left(a_{1}, \ldots, a_{m}\left|c_{1}, \ldots\right| 0, b_{1}, \ldots\right) \\
& =Z\left(a_{1}, \ldots, a_{m}, 0\left|b_{1}, \ldots\right| c_{1}, \ldots\right) .
\end{aligned}
$$

Starting with

$$
\zeta\left(a_{1}, \ldots, a_{m}\right) \zeta\left(b_{1}, \ldots, b_{n}\right)=Z\left(0\left|a_{1}, \ldots, a_{m}\right| b_{1}, \ldots, b_{n}\right),
$$

and applying continually (4.29) and (4.30), we end up with terms of the form

$$
\begin{aligned}
Z\left(a_{1}, \ldots, a_{m}|0| b_{1}, \ldots, b_{n}\right) & =Z\left(a_{1}, \ldots, a_{m}\left|b_{1}, \ldots, b_{n}\right| 0\right) \\
& =\zeta\left(a_{1}, \ldots, a_{m}, b_{1}, \ldots, b_{n}\right) .
\end{aligned}
$$


The resulting identities have the same form as the shuffle identities in the literature, but here advantageously the binomial coefficients in (4.29) explicitly encode (in part) the combinatorics implicit in the shuffle algebra.

Additional multiple $\zeta$ identities can be derived using the three-point identity (3.14). At low lengths and levels it turns out that the multiple $\zeta$ identities obtained in this way are not independent from the set of equations generated by the propagator reversions and partial integrations. Whether this property holds true in general we do not know.

5. Discussion. In the present work we have established a novel representation of multiple $\zeta$ sums in terms of Feynman diagrams in a $1+0$ dimensional quantum field theory. We demonstrated the usefulness of this representation for the derivation of identities between such sums. The encoding into Feynman diagrams proposed here provides a very convenient book-keeping device for certain formal manipulations performed on such sums, as our examples should have amply demonstrated.

Concerning the novelty of the identities derived here, the length-two identities presented in Section 4.1 are, of course, well known. At length three, the identities derived by the partial integration procedure, (4.18) and (4.19), are similar, and presumably equivalent, to the decomposition equations derived in [9] by explicit series manipulations. Similarly, the permutation equations, equation (1) [9, equation (2)] coincide with (4.9) in this paper. Equation (4.12) is contained as a special case in [21, Theorem 2.2]. However, we have not been able to locate in the literature an exact equivalent of our length $m$ identities (4.24) and (4.27). (The special case obtained by combining (4.25) and (4.26) is [21, Theorem 5.1].) The only identities available for arbitrary lengths and levels are those based on the shuffle algebra $[6,7,8,10,26]$ and its generalisations [23]. Of those the depth-length shuffle identities (which are also called stuffle identities or $*$ products [22]) are obviously related, and in fact equivalent to our permutation identities, as we have convinced ourselves. Similarly the weight-length shuffle identities are clearly related to our various partial integration identities. In this case, the question of equivalence is more difficult and requires further investigation.

Note that we did not make use at all of the precise form of the path integral action. Our considerations required the presence of all propagators $g^{(k)}$, as well as of all the vertices $\mathscr{V}^{p, q}$, however they did not determine the statistical weights with which they should appear in the Feynman diagrams. Our choice of the weights for the propagators is mainly motivated by the fact that it leads to a suggestive form for the free path integral determinant. Namely, a simple application of the "In det $=\operatorname{tr} l n$ " identity shows that, formally,

$$
\ln Z(0, \lambda)=\text { const }+\sum_{n=1}^{\infty} \lambda^{n} \frac{\zeta(n)}{n} .
$$

(This calculation may be seen as a chiral generalisation of the calculation of the Scalar QED Euler-Heisenberg Lagrangian performed in [36].) Comparing this expression with the well-known formula for the logarithm of the $\Gamma$ function

$$
\Gamma(1+x)=\exp \left[-\gamma x+\sum_{n=2}^{\infty} \frac{(-1)^{n}}{n} \zeta(n) x^{n}\right],
$$


we see that we can identify the free partition function with the $\Gamma$ function under the assumption that the ill-defined $\zeta(1)$ appearing in (5.1) is renormalised to Euler's constant $\gamma$,

$$
Z_{\text {renorm }}(0, \lambda)=\text { const } \times \Gamma(1-\lambda)
$$

Considering the identities $\gamma=\lim _{n \rightarrow \infty}\left(\sum_{k=1}^{n}(1 / k)-\ln n\right)$ and $\sum_{k=1}^{\infty}\left(1 / k^{\epsilon}\right)=(1 / \epsilon)+$ $\gamma+\mathrm{O}(\epsilon)$ this assumption seems quite natural. Similarly the total propagator becomes relatively simple. Using the integral representation (2.7) of the polylogarithm, it is easily shown that

$$
p_{12} \equiv \sum_{k=0}^{\infty} \lambda^{k}(2 \pi i)^{k} g_{12}^{(k)}=g_{12}^{(0)}+\frac{z_{12} \lambda}{1-\lambda}{ }_{2} F_{1}\left(1,1-\lambda ; 2-\lambda ; z_{12}\right)
$$

For the interaction term there seems to be no such preferred choice. A question of obvious interest (but equally obvious difficulty) is whether nontrivial interaction potentials $V(\mathfrak{g}, \bar{g})$ exist such that the $\zeta$-model would be exactly solvable.

Recently the following generalisation of Euler-Zagier sums (1.3) has attracted some attention,

$$
\zeta\left(k_{1}, \ldots, k_{m} ; \sigma_{1}, \ldots, \sigma_{m}\right)=\sum_{n_{1}>n_{2}>\cdots>n_{m}>0} \frac{\sigma_{1}^{n_{1}} \cdots \sigma_{m}^{n_{m}}}{n_{1}^{k_{1}} \cdots n_{m}^{k_{m}}}
$$

where $\sigma_{j}= \pm 1$. Those alternating Euler-Zagier sums arise naturally in the calculation of ultraviolet divergences in renormalisable quantum field theories, and in the application of knot theory to the classification of those divergences [13]. At the same time, the inclusion of alternating Euler-Zagier sums seems to simplify the problem of reducing the set of all such sums to a basic set via multiple $\zeta$ identities [10]. (For a tabulation of alternating series see [5].)

More generally, arbitrary $N$ th roots of unity in place of the $\sigma_{j}$ have been considered in connection with the study of mixed Tate motives over Spec $\mathbb{Z}[18,34]$. Those phase factors can be easily accommodated in the $\zeta$-model. To generate a phase factor $\sigma=$ $e^{2 \pi i s}$, the propagator (2.1) has to be simply replaced by

$$
\mathcal{g}_{\sigma}^{(k)}\left(u_{12}\right) \equiv \sum_{n=1}^{\infty} \frac{e^{2 \pi i(n+s) u_{12}}}{(2 \pi i n)^{k}}
$$

On the path integral level this can be achieved by changing from periodic to twisted boundary conditions, $x(1)=\sigma x(0)$, and replacing $\partial$ by $\partial-2 \pi i$.

ACKNOWLEDGMENTS. We would like to thank C. Zeta-Jones for inspiration. Helpful discussions with D. Broadhurst and D. Kreimer are gratefully acknowledged. We also thank M. E. Hoffman for detailed comments on the electronic preprint version of this paper, and L. Dixon for pointing out a typo. One of us (U. Müller) would like to thank the LAPTH for the kind hospitality during a stay when part of this work was done. 


\section{REFERENCES}

[1] T. M. Apostol and T. H. Vu, Dirichlet series related to the Riemann zeta function, J. Number Theory 19 (1984), no. 1, 85-102.

[2] T. Arakawa and M. Kaneko, Multiple zeta values, poly-Bernoulli numbers, and related zeta functions, Nagoya Math. J. 153 (1999), 189-209.

[3] F. Bastianelli and P. van Nieuwenhuizen, Trace anomalies from quantum mechanics, Nuclear Phys. B 389 (1993), no. 1, 53-80.

[4] Z. Bern and D. A. Kosower, The computation of loop amplitudes in gauge theories, Nuclear Phys. B 379 (1992), no. 3, 451-561.

[5] M. Bigotte, G. Jacob, N. E. Oussous, and M. Petitot, Tables des relations de la fonction zéta colorée, Report of the LIFL Publications, IT-322, University Lille I, 1998.

[6] J. M. Borwein, D. M. Bradley, and D. J. Broadhurst, Evaluations of k-fold Euler/Zagier sums: A compendium of results for arbitrary $k$, Electron. J. Combin. 4 (1997), no. 2, 1-21.

[7] J. M. Borwein, D. M. Bradley, D. J. Broadhurst, and P. Lisoněk, Combinatorial aspects of multiple zeta values, Electron. J. Combin. 5 (1998), no. 1, 1-12.

[8] _ Special values of multiple polylogarithms, Trans. Amer. Math. Soc. 353 (2001), no. 3, 907-941.

[9] J. M. Borwein and R. Girgensohn, Evaluation of triple Euler sums, Electron. J. Combin. 3 (1996), no. 1, 1-27.

[10] D. J. Broadhurst, Conjectured enumeration of irreducible multiple zeta values, from knots and Feynman diagrams, preprint OUT-4102-65, 1996, http://arxiv.org/abs/ hep-th/9612012.

[11] _ Solving differential equations for 3-loop diagrams: relation to hyperbolic geometry and knot theory, preprint OUT-4102-74, 1998, http://arxiv.org/abs/ hep-th/9806174.

[12] D. J. Broadhurst, R. Delbourgo, and D. Kreimer, Unknotting the polarized vacuum of quenched QED, Phys. Lett. B 366 (1996), 421.

[13] D. J. Broadhurst and D. Kreimer, Association of multiple zeta values with positive knots via Feynman diagrams up to 9 loops, Phys. Lett. B 393 (1997), no. 3-4, 403-412.

[14] J. de Boer, B. Peeters, K. Skenderis, and P. van Nieuwenhuizen, Loop calculations in quantum mechanical non-linear sigma models with fermions and applications to anomalies, Nuclear Phys. B 459 (1996), no. 3, 631-692.

[15] V. G. Drinfel'd, On quasitriangular quasi-Hopf algebras and a group closely connected with $\operatorname{Gal}(\overline{\mathbb{Q}} / \mathbb{Q})$, Leningrad Math. J. 2 (1991), no. 4, 829-860.

[16] L. Euler, Meditationes circa singulare serierum genus, Novi Comm. Acad. Sci. Petropol. 20 (1775), 140-186.

[17] H. Furusho, The multiple zeta value algebra and the stable derivation algebra, Sūrikaisekikenkyūsho Kōkyūroku (2001), no. 1200, 137-148.

[18] A. B. Goncharov, Polylogarithms in arithmetic and geometry, Proceedings of the International Congress of Mathematicians, Vol. 1, 2 (Zürich, 1994), Birkhäuser, Basel, 1995, pp. 374-387.

[19] _ Multiple polylogarithms, cyclotomy and modular complexes, Math. Res. Lett. 5 (1998), no. 4, 497-516.

[20] A. Granville, A decomposition of Riemann's zeta-function, Analytic Number Theory (Kyoto, 1996) (Y. Motohashi, ed.), London Mathematical Society Lecture Note Series, vol. 247, Cambridge University Press, Cambridge, 1997, pp. 95-101.

[21] M. E. Hoffman, Multiple harmonic series, Pacific J. Math. 152 (1992), no. 2, 275-290.

[22] _ The algebra of multiple harmonic series, J. Algebra 194 (1997), no. 2, 477-495.

[23] _ Quasi-shuffle products, J. Algebraic Combin. 11 (2000), no. 1, 49-68.

[24] M. E. Hoffman and C. Moen, Sums of triple harmonic series, J. Number Theory 60 (1996), no. 2, 329-331.

[25] M. E. Hoffman and Y. Ohno, Relations of multiple zeta values and their algebraic expression, preprint, 2000, http://arxiv.org/abs/math.QA/0010140. 
[26] C. Kassel, Quantum Groups, Graduate Texts in Mathematics, vol. 155, Springer-Verlag, New York, 1995.

[27] D. Kreimer, Knots and divergences, Phys. Lett. B 354 (1995), no. 1-2, 117-124.

[28] T. Q. T. Le and J. Murakami, Kontsevich's integral for the Homfly polynomial and relations between values of multiple zeta functions, Topology Appl. 62 (1995), no. 2, 193206.

[29] L. Lewin, Polylogarithms and Associated Functions, North-Holland Publishing, New York, 1981.

[30] L. Lewin (ed.), Structural Properties of Polylogarithms, American Mathematical Society, Rhode Island, 1991.

[31] C. Markett, Triple sums and the Riemann zeta function, J. Number Theory 48 (1994), no. 2, 113-132.

[32] Y. Ohno, A generalization of the duality and sum formulas on the multiple zeta values, J. Number Theory 74 (1999), no. 1, 39-43.

[33] A. M. Polyakov, Gauge Fields and Strings, Harwood Academic, Chur, 1987.

[34] G. Racinet, Torseurs associés à certaines relations algébriques entre polyzêtas aux racines de l'unité [Torsor structure associated to some algebraic relations between polyzetas at roots of unity], C. R. Acad. Sci. Paris Sér. I Math. 333 (2001), no. 1, 5-10 (French).

[35] R. S. Rao and M. V. Subbarao, Transformation formulae for multiple series, Pacific J. Math. 113 (1984), no. 2, 471-479.

[36] M. G. Schmidt and C. Schubert, On the calculation of effective actions by string methods, Phys. Lett. B 318 (1993), no. 3, 438-446.

[37] _ Multiloop calculations in the string inspired formalism: The single spinor loop in QED, Phys. Rev. D 53 (1996), no. 4, 2150-2159.

[38] C. Schubert, An introduction to the worldline technique for quantum field theory calculations, Acta Phys. Polon. B 27 (1996), 3965-4001.

[39] V. A. Smirnov, Renormalization and Asymptotic Expansions, Birkhäuser Verlag, Basel, 1991.

[40] M. J. Strassler, Field theory without Feynman diagrams: one-loop effective actions, Nuclear Phys. B 385 (1992), no. 1-2, 145-184.

[41] T. Terasoma, Mixed Tate motives and multiple zeta values, preprint, 2001, http://arxiv.org/abs/math.NT/0104231.

[42] _ Selberg integral and multiple zeta values, preprint, 1999, http://arxiv.org/abs/ math.AG/9908045.

[43] L. Tornheim, Harmonic double series, Amer. J. Math. 72 (1950), 303-314.

[44] D. Zagier, Values of zeta functions and their applications, First European Congress of Mathematics, Vol. II (Paris, 1992), Progress in Mathematics, vol. 120, Birkhäuser, Basel, 1994, pp. 497-512.

UWE MÜLlER: InSTITUT FÜR PHYSIK, JOHANNES-GUTENBERG-UNIVERSITÄT MAINZ, STAUDINGER-WEG 7, D-55099 MAINZ, GERMANY

E-mail address: umue11er@thep. physik.uni-mainz.de

Christian Schubert: Laboratoire D’AnNecy-Le-Vieux De Physique Théorique LAPTH, Chemin de Bellevue, BP 110, F-74941 ANNECY-Le-VIeux CEDEX, FrANCE

E-mail address: schubert@1app.in2p3.fr 


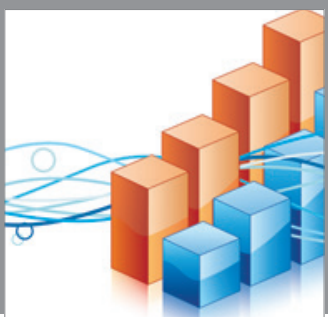

Advances in

Operations Research

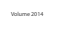

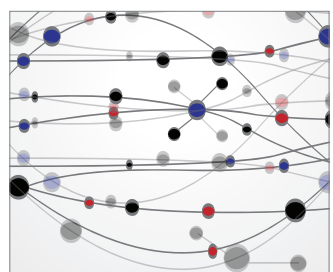

\section{The Scientific} World Journal
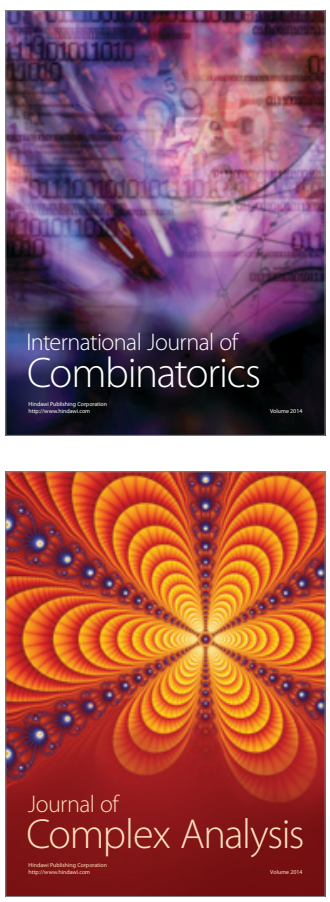

International Journal of

Mathematics and

Mathematical

Sciences
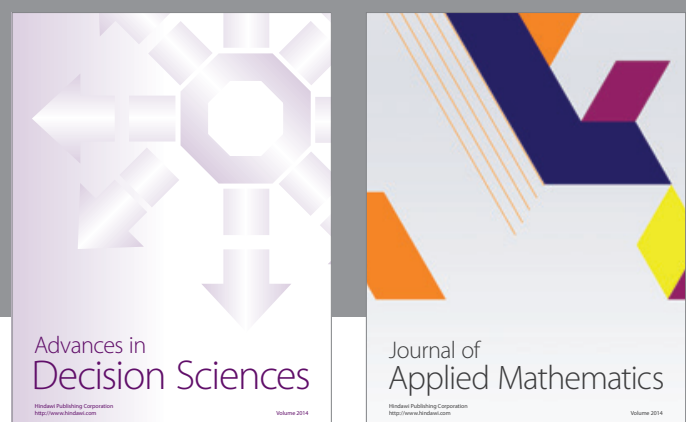

Journal of

Applied Mathematics
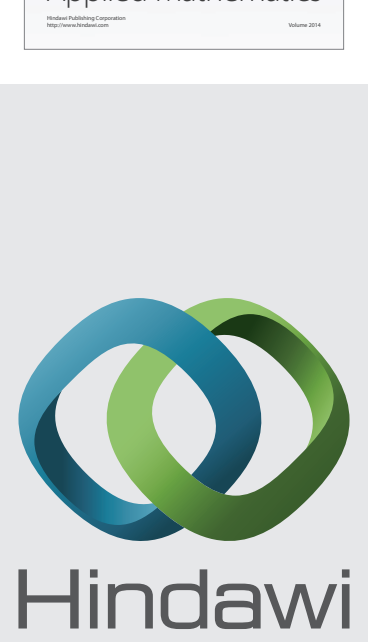

Submit your manuscripts at http://www.hindawi.com
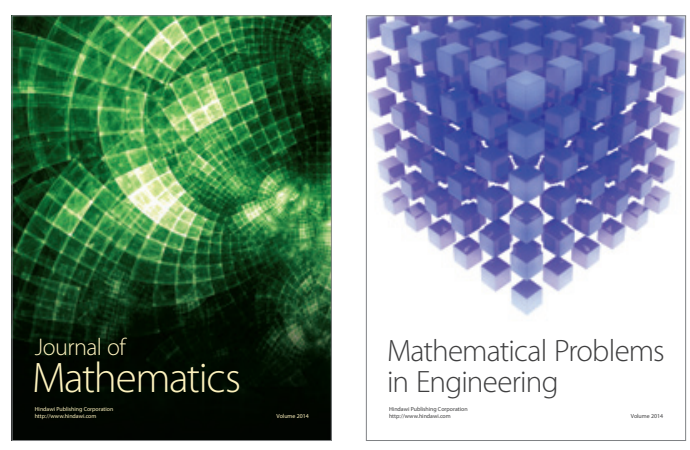

Mathematical Problems in Engineering
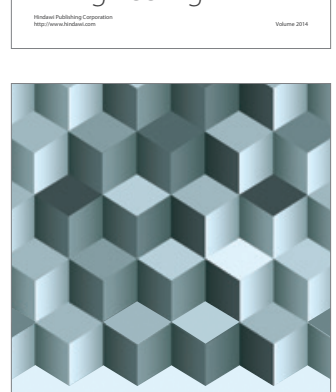

Journal of

Function Spaces
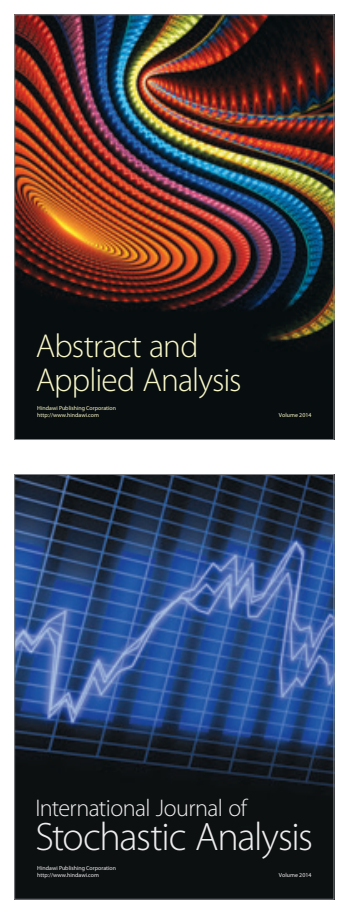

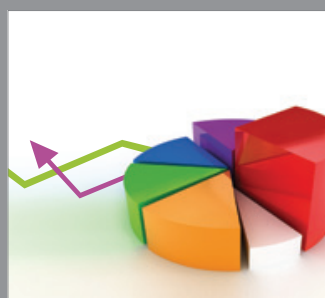

ournal of

Probability and Statistics

Promensencen
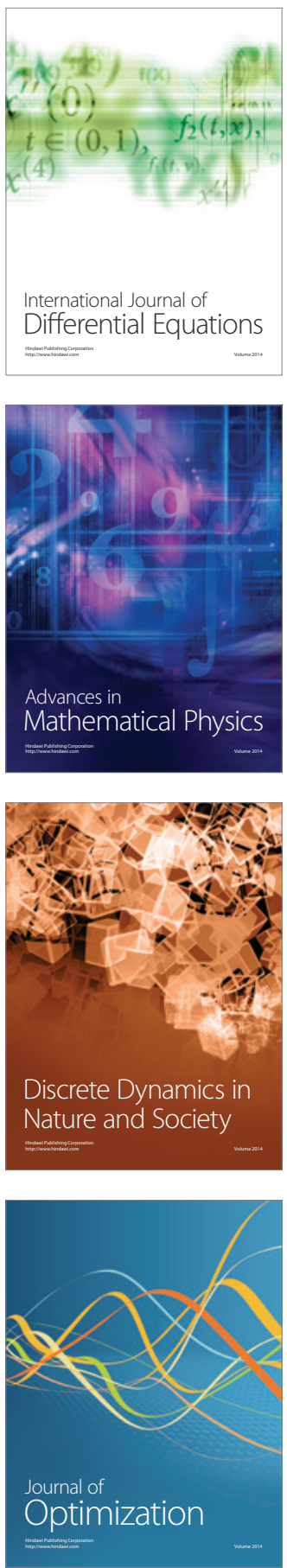